\title{
Anaplastic lymphoma kinase-negative anaplastic large cell lymphoma with colon involvement
}

\author{
Yuko Sakakibara MD¹, Shoichi Nakazuru MD¹, Takuya Yamada MD¹, Tetsuya Iwasaki MD¹, Ryuichiro Iwasaki MD¹, \\ Akio Ishihara $M D^{1}$, Kumiko Nishio $M D^{1}$, Hisashi Ishida $M D^{1}$, Yoshinori Kodama $M D^{2}$, Eiji Mita MD $^{1}$
}

\section{CASE PRESENTION}

$\mathrm{A}_{\mathrm{i}}$ 65-year-old man was referred to the authors' hospital for examination of a painful hard mass in the left buttock. Magnetic resonance imaging revealed a mass in the left ilium, and ${ }^{18} \mathrm{~F}$-fluoro-2deoxy-glucose positron emission tomography/computed tomography revealed uptake in the left ilium and ascending colon. Colonoscopy revealed a reddish ulcerative lesion with protrusions in the ascending colon (Figure 1A). Endoscopy with dual-focus narrow-band imaging revealed dilated, tortuous microvessels and avascular areas in the protrusions (Figure 1B). Histopathological examination of biopsy specimens revealed infiltration of large lymphoid cells with immunohistological characteristics similar to those of the iliac tumour (Figure 2A). Immunohistochemical staining was positive for CD30 (Figure 2B) and negative for anaplastic lymphoma kinase (ALK). Based on these findings, the patient was diagnosed with ALK-negative anaplastic large cell lymphoma (ALCL) with colon involvement. He received six cycles of CHOP (cyclophosphamide, doxorubicin, vincristine and prednisone) chemotherapy and achieved complete remission.

\section{DISCUSSION}

ALCL is a CD30-positive T cell neoplasm; systemic-type ALCL represents $2 \%$ to $3 \%$ of all non-Hodgkin lymphomas and $12 \%$ to $14 \%$ of $\mathrm{T}$ cell non-Hodgkin lymphomas $(1,2)$. The WHO classification system divides ALCLs into ALK-positive and ALK-negative groups. Systemic ALK-negative ALCL patients have a worse prognosis than ALK-positive ALCL patients, with five-year survival rates of $49 \%$ and $70 \%$, respectively (1). ALK-negative ALCL involves both lymph nodes and extranodal sites (20\% of cases) (2). The most frequent extranodal involvement sites are the skin, lungs, liver and gastrointestinal tract, whereas colon involvement is extremely rare $(1,2)$. To our knowledge, only two previous case reports presented endoscopic findings of ALCL with colon involvement; one case showed multiple elevated lesions with ulceration at the apex, and the other showed ulcerated stricture $(3,4)$. If ulcerative colonic lesions are observed on colonoscopy, colon involvement of ALCL should be considered as a rare differential diagnosis.

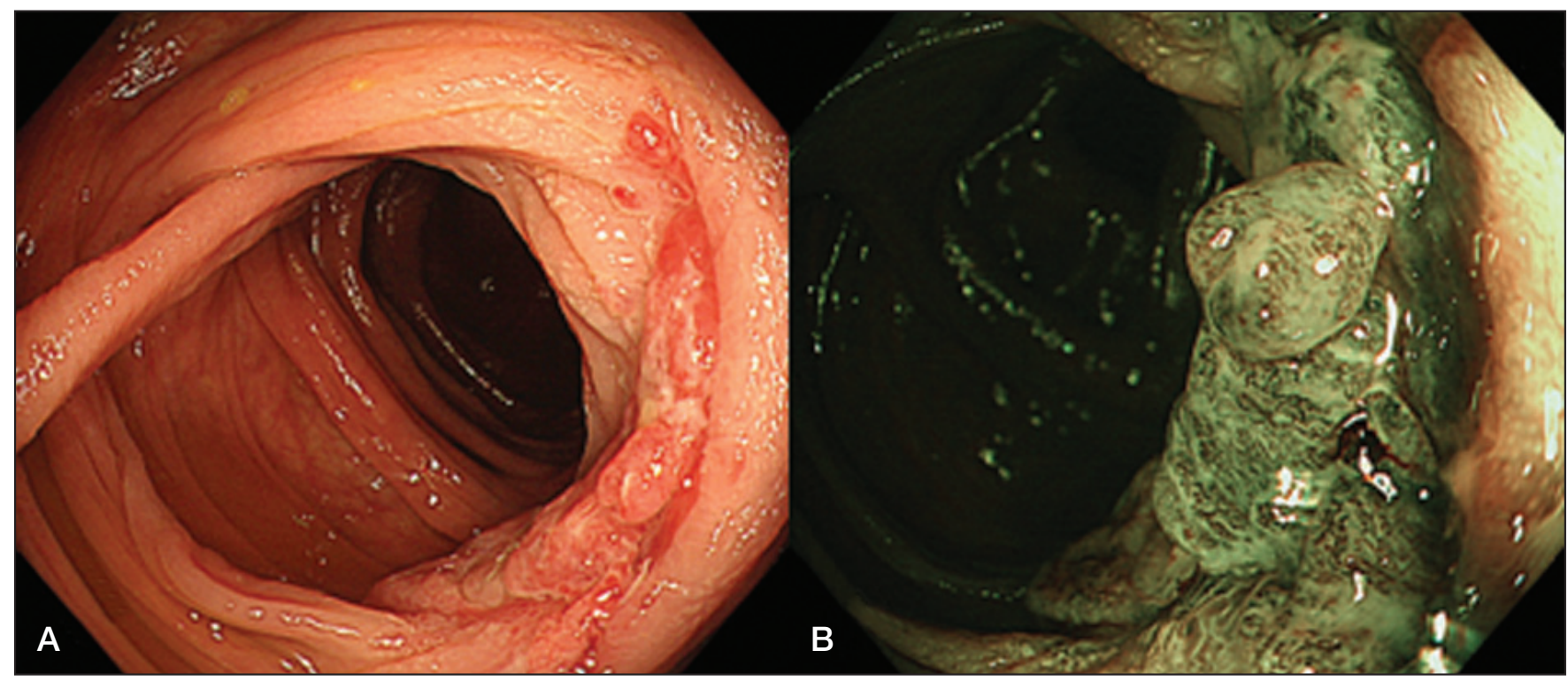

Figure 1) A Endoscopic image of the ascending colon showing a reddish ulcerative lesion with protrusions. B Endoscopy with dual-focus narrow-band imaging revealing dilated, tortuous microvessels and avascular areas in the protrusions

${ }^{1}$ Department of Gastroenterology and Hepatology; ${ }^{2}$ Department of Pathology, National Hospital Organization, Osaka National Hospital, Osaka, Japan Correspondence and reprints: Dr Yuko Sakakibara, Department of Gastroenterology and Hepatology, National Hospital Organization, Osaka National

Hospital, 2-1-14 Houenzaka, Chuo-ku, Osaka city, Osaka 540-0006, Japan. Telephone 81-6-6942-1331, fax 81-6-6946-3569,

e-mail yuko.s@onh.go.jp

Received for publication June 3, 2015. Accepted June 5, 2015 


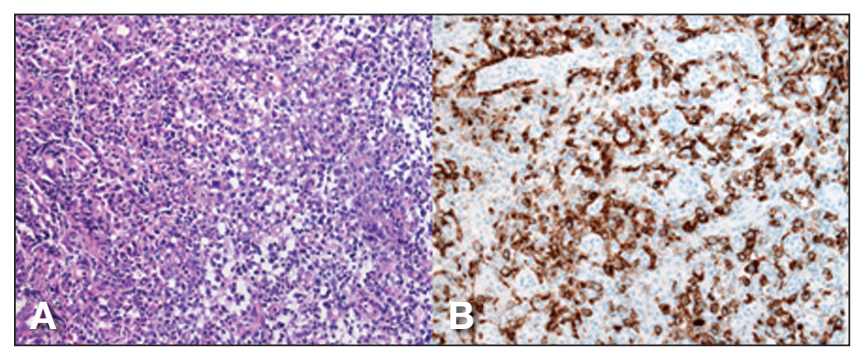

Figure 2) A Photomicrograph of the biopsy specimen from the ascending colonic lesion shows infiltration of large lymphoid cells (hematoxylin and eosin stain, original magnification $\times 20$ ). B Immunohistochemical staining showing large lymphoid cells strongly positive for CD30

\section{REFERENCES}

1. Savage KJ, Harris NL, Vose JM, et al. ALK- anaplastic large-cell lymphoma is clinically and immunophenotypically different from both ALK+ ALCL and peripheral T-cell lymphoma, not otherwise specified: Report from the International Peripheral T-Cell Lymphoma Project. Blood 2008;111:5496-504.

2. Ferreri AJ, Govi S, Pileri SA, et al. Anaplastic large cell lymphoma, ALK-negative. Crit Rev Oncol Hematol 2013;85:206-15.

3. Matsumoto H, Koga H, Honda K, et al. Characterization of secondary GI lesions with anaplastic large-cell $(\mathrm{Ki}-1)$ lymphoma: A first report of two cases. Gastrointest Endosc 2005;61:607-9.

4. Shukla T, Jin J, Marginean EC, et al. Anaplastic large cell lymphoma of the colon in a patient with colonic Crohn disease treated with infliximab and methotrexate. Can J Gastroenterol Hepatol 2014;28:11-2.

The Canadian Journal of Gastroenterology $\mathcal{E}$ Hepatology is considering a limited number of submissions for IMAGE OF THE MONTH. These are based on endoscopic, histological, radiological and/or patient images, which must be anonymous with no identifying features visible. The patient must consent to publication and the consent must be submitted with the manuscript. All manuscripts should be practical and relevant to clinical practice, and not simply a case report of an esoteric condition. The text should be brief, structured as CASE PRESENTATION and DISCUSSION, and not more than 700 words in length. A maximum of three images can be submitted and the number of references should not exceed five. The submission may be edited by our editorial team. 


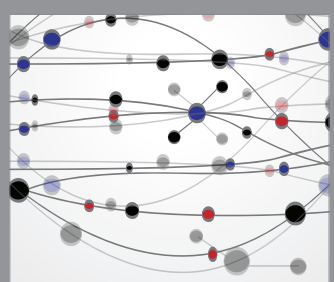

The Scientific World Journal
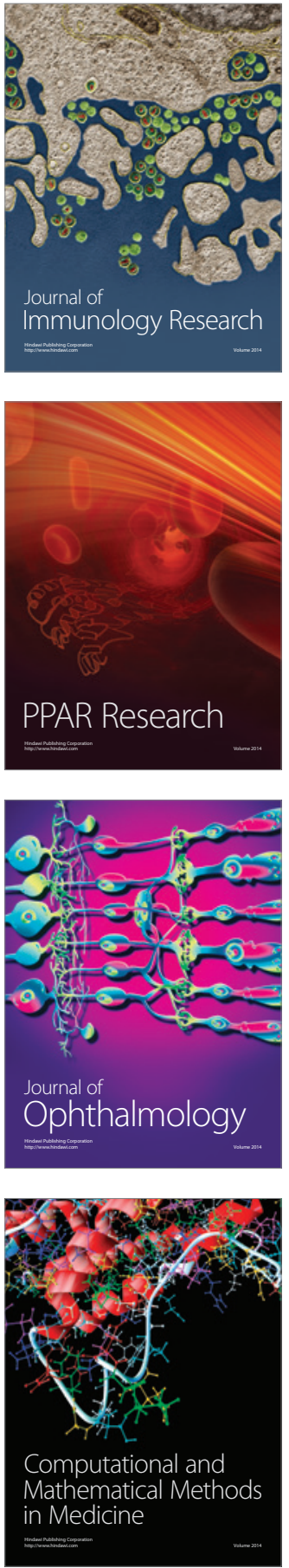

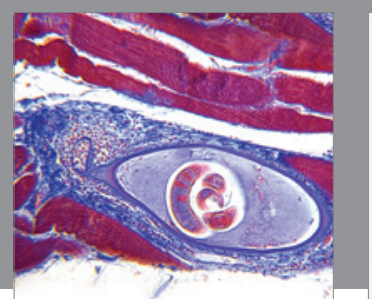

Gastroenterology Research and Practice

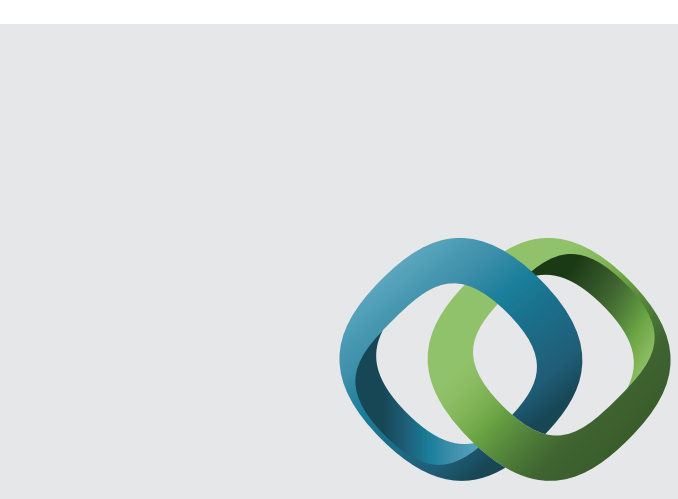

\section{Hindawi}

Submit your manuscripts at

http://www.hindawi.com
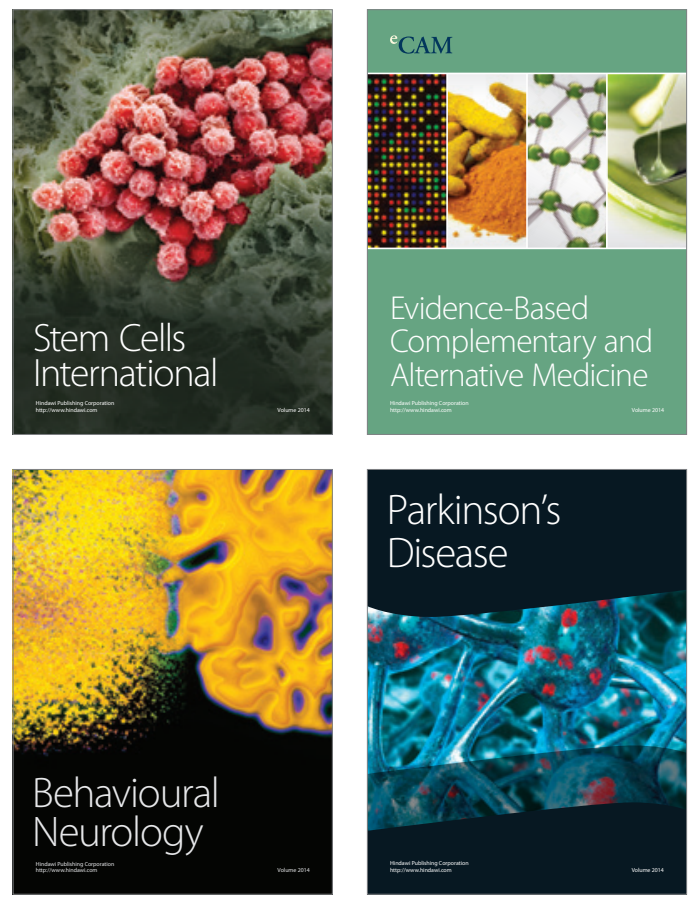
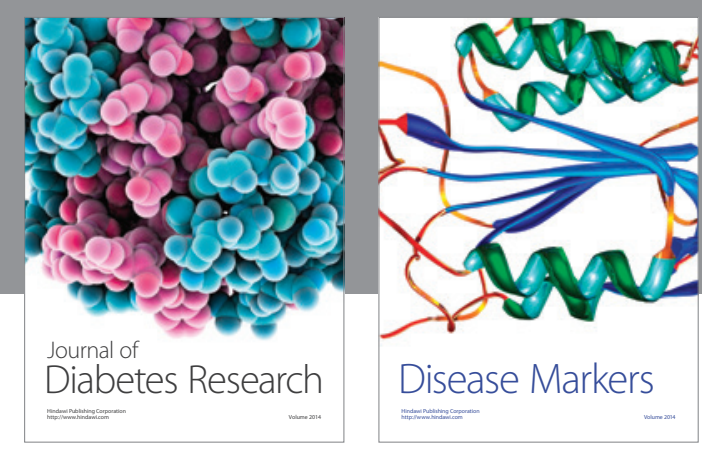

Disease Markers
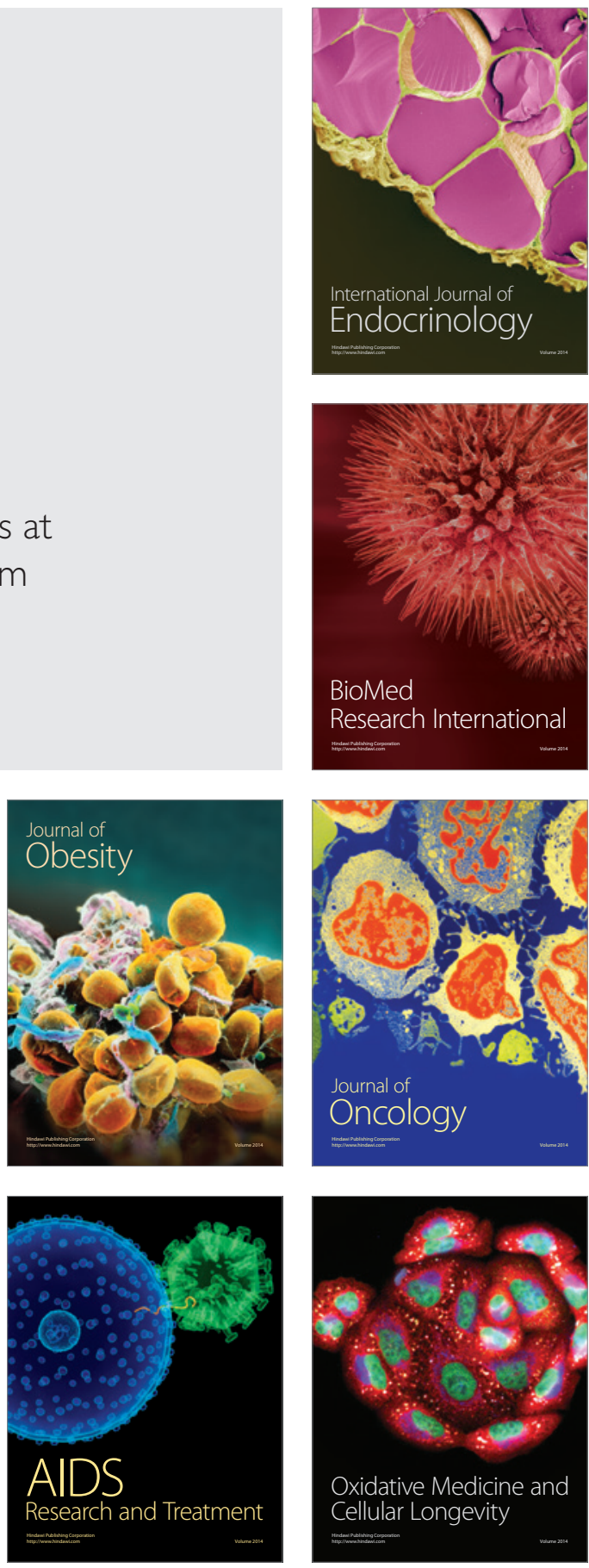